Pacific Journal of Mathematic 


\section{AN INTRINSIC CHARACTERIZATION FOR PI FLOWS}

\section{Douglas C. McMahon and Louis J. Nachman}

An intrinsic characterization for a minimal flow $(X, T)$ to be a PI flow is given. This characterization is then combined with some recent techniques of $R$. Ellis to prove the general PI and HPI versions of the Veech Structure Theorem.

o. Introduction. A pointed minimal flow $\left(X, x_{0}, T\right)$ is PI if there is an ordinal $\Lambda$, a collection of pointed minimal flows $\left\{\left(X_{\lambda}, x_{\lambda}\right)\right.$ : $\lambda \leqq \Lambda\}$, and a homomorphism $\pi, \pi:\left(X_{1}, x_{\Lambda}\right) \rightarrow\left(X, x_{0}\right)$ such that

(i) $X_{0}$ is the trivial flow

(ii) for each $\lambda<\Lambda$ there is a homomorphism $\varphi_{\lambda}^{\lambda+1}, \varphi_{\lambda}^{++1}:\left(X_{\lambda+1}, x_{\lambda+1}\right) \rightarrow$ $\left(X_{\lambda}, x_{\lambda}\right)$ which is either proximal or almost periodic,

(iii) for each limit ordinal $\lambda_{0} \leqq \Lambda,\left(X_{\lambda_{0}}, x_{\lambda_{0}}\right)$ is $\operatorname{inv} \lim \left\{\left(X_{\lambda}, x_{\lambda}\right)\right.$ : $\left.\lambda<\lambda_{0}\right\}$, and

(iv) $\pi$ is proximal.

The collection of flows $\left\{\left(X_{\lambda}, x_{\lambda}\right): \lambda \leqq \Lambda\right\}$ and the associated maps $\left\{\varphi_{\lambda}^{\lambda+1}\right\}_{\lambda<\Lambda}$ are called a PI tower for $\left(X, x_{0}, T\right) .\left(X, x_{0}, T\right)$ is strictly PI if $\pi$ is the identity map. For a discussion of the role of PI flows in topological dynamics, see part 2 of Veech's article [9].

With the exception of the definition, the only condition equivalent to PI in the literature is that the group for the flow, $G(X$, $x_{0}$ ), contain the group $G_{\infty}$. (See [10] for a characterization of PD flows.) In this paper we give an intrinsic characterization of PI. Section 1 is devoted to this characterization.

In a recent paper, [3], Ellis proved that the Furstenberg structure theorem holds for any distal flow. Modifying Ellis's technique and applying our characterization we show, in $\S 2$, that for a large class of properties of flows, all flows with one of these properties are PI iff all metric flows with the same property are PI. As a corollary, we show that every point-distal flow is PI. Using this fact we establish that every point-distal flow is actually HPI (the proximal maps in the PI tower are highly proximal) thus proving the General Veech Structure Theorem.

We assume throughout this paper that the reader is familiar with the general theory of PI flows as contained in [5] or [6]. Our notation is primarily that of Glasner's book, [6], with the obvious modification that our group actions are written on the right. In particular, for a fixed topological group $T, M(T)$, or just $M$, is a fixed minimal right ideal in $\beta T$ with the usual semi-group structure. $J(T)$, or just $J$, is the set of idempotents in $M(T)$. If $U \subseteq T$ then 
$[U, U]=\{p \in M(T) \mid$ there is a $t \in U$ such that $p t \in \bar{U}\}$ where $\bar{U}$ is the closure of $U$ in $\beta T$.

If $\left(X, x_{0}\right)$ and $\left(Y, y_{0}\right)$ are pointed minimal sets and $\varphi:\left(X, x_{0}\right) \rightarrow$ $\left(Y, y_{0}\right)$ then $R(\varphi)=\left\{\left(x_{1}, x_{2}\right) \in X \times X \mid \varphi\left(x_{1}\right)=\varphi\left(x_{2}\right)\right\}, Q(\varphi)$ is the relative regionally proximal relation, $S(\varphi)$ is the relative equicontinuous structure relation, $D(\varphi)$ is the set of almost periodic points in $R(\varphi)$. $G\left(X, x_{0}\right)$ and $G\left(Y, y_{0}\right)$ are the groups for $\left(X, x_{0}\right)$ and $\left(Y, y_{0}\right)$ relative to a fixed $u \in J(T)$. We denote the map from $\beta T$ to $X$ given by $p \rightarrow x_{0} p$ by $e_{x_{0}}$. We use $c_{X}$, or just $c$ to denote closure in $X$.

We wish to 'thank the referee for pointing out an error in an earlier version of Lemma 1.1 of this paper and for suggesting shorter proofs of (1) implies (2) of Theorem 1.1 and of Lemma 2.3.

1. PI Flows. The characterization of PI flows we present involves the nice behavior of a certain class of closed subsets of the flow. The nice behavior involves a relation which looks like a strong localization of the regionally proximal relation. (See [9] where a nonlocal version of this relation is mentioned.) To be specific, if $(X, T)$ is a flow, $K$ a subset of $X$, and $x$ and $y$ belong to $K$, we say $x$ is strongly regionally proximal to $y$ in $K$, which we abbreviate $x \in S R P(K, y)$, if there are nets $\left\{k_{n}\right\}$ in $K$ and $\left\{t_{n}\right\}$ in $T$ such that $\lim k_{n}=y, \lim k_{n} t_{n}=x$, and $\lim x t_{n}=x$.

The easiest way to describe the class of closed subsets we are interested in is by using $J(T)=J$. The closed subsets we need are those sets $K$ contained in $X$ which contain at least two points (we will call them nontrivial) and for which $K w$ is dense in $K$ for some $w \in J$.

The referee noted that by 2.2 of [5], if $x, y \in K u$, then $x \in$ $S R P(K u, y)$ iff $y$ is in the $X$ closure of $K u \cap U$ for every $F$-neighborhood $U$ of $x$. In particular, $x \in \operatorname{SRP}(K u, y)$ implies $y$ is in the $F$ closure of $K u \cap U$ for every $F$-neighborhood $U$ of $x$. A proof of (5) implies (1) in the following theorem could be given by exploiting these ideas.

THEOREM 1.1. Suppose $(X, T)$ is minimal. Then the following are equivalent:

(1) $(X, T)$ is not PI.

(2) For some $w \in J$ there is a closed, nontrivial set $K \subseteq X$ such that $K=c_{X}(K w)$ and such that for each $x \in K, x \in \operatorname{SRP}(K, y)$ for all $y \in K$.

(3) For some $w \in J$ there is a closed, nontrivial set $K \cong X$ such that $K=c_{X}(K w)$ and such that for some $x \in K, x \in \operatorname{SRP}(K, y)$ for all $y \in K$. 
(4) For some $w \in J$ there is a closed, nontrivial set $K \cong X$ such that $K=c_{X}(K w \cap K)$ and such that for each $x \in K, x \in \operatorname{SRP}(K$, y) for all $y \in K$.

(5) For some $w \in J$ there is a closed, nontrivial set $K \cong X$ such that $K=c_{X}(K w \cap K)$ and such that for some $x \in K, x \in \operatorname{SRP}(K$, y) for all $y \in K$.

Proof. Clearly (2) implies (3), (2) implies (4), (4) implies (5), and (3) implies (5). We show that (1) implies (2) and that not (1) implies not (5).

(1) implies (2).

Throughout this proof we use the notation of [6] with the modification mentioned in the introduction and all references are to [6].

Let $(X, T)$ be a flow which is not PI. Let $M$ be the universal minimal flow and let $u \in J$. Consider $G_{\infty}(u)=G_{\infty}$ for the pointed minimal flow $(M, u)$ as defined on pages 135, 138. Fix $u \in J$, let $F=G_{\infty}(u)$, and let $w \in J$ for which $F w$ is an $F$ minimal flow with $F$ acting on the left (see page 142). Consider $(M, w)$ and note that $G_{\infty}(w)=F w$. Applying 6.2, page 143, with $u$ replaced by $w, F$ by $G_{\infty}(w)$, and $A=\{w\}$, and noting that $H\left(G_{\infty}(w)\right)=G_{\infty}(w)$, we have that, for each neighborhood $U$ of $w$, the set $\{p \in M: w t \in U$ and $p t \in U\}$ is dense in $c_{M}\left(G_{\infty}(w)\right)$. Now suppose $p \in c_{M}\left(G_{\infty}(w)\right)$, then $p c_{M}\left(G_{\infty}(w)\right)=p w c_{M}\left(G_{\infty}(w)\right)=c_{M}\left(p w G_{\infty}(w)\right)=c_{M}\left(G_{\infty}(w)\right)$ since $p w \in G_{\infty}(w)$ by Lemma 1.5, page 115.

Now fix $x_{0}$ in $X$ with $x_{0} w=x_{0}$ and take $K=x_{0} c_{M}\left(G_{\infty}(w)\right)$ which is $c_{X}\left(x_{0} G_{\infty}(w)\right)$. Let $x \in K$ and note that $x c_{M}\left(G_{\infty}(w)\right)=K$. Then clearly $x \in \operatorname{SRP}(K, y)$ for all $y \in K$. Finally note that $K w$ is a dense subset of $K$ and thus (1) implies (2).

Not (1) implies not (5).

Let $(X, T)$ be PI and let $\left\{X_{\lambda}: \lambda \leqq \Lambda\right\}$ and $\left\{\varphi_{\lambda}^{\lambda+1}\right\}_{\lambda<\Lambda}$ be a PI tower for $(X, T)$. Let $\pi:\left(X_{\Lambda}, T\right) \rightarrow(X, T)$ be the proximal map. We will use $c_{\lambda}$ for closure in $X_{\lambda}, c$ for closure in $X$. Let $w$ be any element of $J$ and let $K$ be any nontrivial closed set in $X$ such that $c(K w \cap K)=K$. (Note if $c(K u \cap K)$ is trivial for all $K \subseteq X$, then Not (1) implies Not (5) immediately). Let $K^{*}=c_{\Lambda}\left(\pi^{-1}[K] w \cap \pi^{-1}[K]\right)$. Note that $\pi$ maps $K^{*}$ onto $K$.

For each $\lambda<\Lambda$, let $\varphi_{\lambda}: X_{\Lambda} \rightarrow X_{\lambda}$ be the obviously induced map. Let $\beta<\Lambda$ be the smallest ordinal for which $\varphi_{\beta}\left(K^{*}\right)$ is not a single point. We note that, because of condition (iii) of the definition of PI flows, $\beta$ is not a limit ordinal. Therefore, $\beta-1$ is also an ordinal. 
We first show that $\varphi_{\beta-1}^{\beta}: X_{\beta} \rightarrow X_{\beta-1}$ is not a proximal map. To see this, note that if it were, all points in $\varphi_{\beta}\left(K^{*}\right)$ would be proximal since $\varphi_{\beta-1}^{\beta} \varphi_{\beta}\left(K^{*}\right)$ is a single point. Since $\pi^{-1}[K] w \cap \pi^{-1}[K]$ is dense in $K^{*}, \varphi_{\beta}\left(\pi^{-1}[K] w \cap \pi^{-1}[K]\right)$ is dense in $\varphi_{\beta}\left(K^{*}\right)$. Let $x^{*}$ and $y^{*}$ be in $\pi^{-1}[K] w \cap \pi^{-1}[K]$. Then $x^{*}, y^{*} \in \pi^{-1}[K] w$ and therefore $x^{*} w=x^{*}$ and $y^{*} w=y^{*}$. Thus $\varphi_{\beta}\left(x^{*}\right) w=\varphi_{\beta}\left(x^{*}\right)$ and $\varphi_{\beta}\left(y^{*}\right) w=\varphi_{\beta}\left(y^{*}\right)$. So, if all points in $\varphi_{\beta}\left(K^{*}\right)$ are proximal, $\varphi_{\beta}\left(x^{*}\right)=\varphi_{\beta}\left(y^{*}\right)$ and $\varphi_{\beta}\left(K^{*}\right)$ has a dense subset of only one point, contradicting the choice of $\beta$. Since $\phi_{\beta-1}^{\beta}$ is not proximal, it must be almost periodic.

We show that $K$ does not satisfy (5) of the statement of the theorem by demonstrating that for every $x \in K$ there is a $y \in K$ such that $x \notin S R P(K, y)$. With this in mind, let $x \in K$ and choose $x^{*} \in K^{*}$ such that $\pi\left(x^{*}\right)=x$. Let $y^{*}$ be any element of $K^{*}$ such that $\varphi_{\beta}\left(y^{*}\right) \neq \varphi_{\beta}\left(x^{*}\right)$ and let $y=\pi\left(y^{*}\right)$. If $x$ and $y$ are equal, then $x^{*}$ and $y^{*}$ are proximal and thus $\varphi_{\beta}\left(x^{*}\right)$ and $\varphi_{\beta}\left(y^{*}\right)$ are proximal. But $\varphi_{\beta-1}^{\beta}$ is almost periodic and hence does not identify any proximal points. Therefore, since $\varphi_{\beta}\left(x^{*}\right) \neq \varphi_{\beta}\left(y^{*}\right)$ and $\varphi_{\beta-1}^{\beta} \varphi_{\beta}\left(x^{*}\right)=\phi_{\beta-1}^{\beta} \varphi_{\beta}\left(y^{*}\right)$, we conclude that $x \neq y$.

Now suppose $x \in \operatorname{SRP}(K, y)$. Then we can find nets $\left\{k_{n}\right\}$ in $K$ and $\left\{t_{n}\right\}$ in $T$ such that $\lim k_{n}=y$ and $\lim \left(x, k_{n}\right) t_{n}=(x, x)$. For each $n$, let $k_{n}^{*} \in K^{*}$ such that $\pi\left(k_{n}^{*}\right)=k_{n}$. Since $X_{A}$ is compact there are subnets $\left\{k_{m}^{*}\right\}$ and $\left\{t_{m}\right\}$ such that the three nets $\left\{k_{m}^{*}\right\},\left\{k_{m}^{*} t_{m}\right\}$, and $\left\{x^{*} t_{m}\right\}$ all converge, say to $z^{*}, b^{*}$, and $a^{*}$ respectively. Since $\pi$ is proximal, $z^{*}$ and $y^{*}$ are proximal, and $a^{*}, b^{*}$, and $x^{*}$ are proximal. Now, since $\varphi_{\beta-1}\left(k^{*}\right)$ is a singleton, $\varphi_{\beta-1}\left(x^{*}\right)=\varphi_{\beta-1}\left(k_{m}^{*}\right)$ and $\varphi_{\beta-1}\left(x^{*} t_{m}\right)=$ $\varphi_{\beta-1}\left(k^{*} t_{m}\right)$. Thus $\varphi_{\beta-1}\left(x^{*}\right)=\varphi_{\beta-1}\left(y^{*}\right)$ and $\varphi_{\beta-1}\left(a^{*}\right)=\varphi_{\beta-1}\left(b^{*}\right)$. Therefore, since $\varphi_{\beta-1}$ is almost periodic, $\varphi_{\beta}\left(x^{*}\right)=\varphi_{\beta}\left(y^{*}\right)$ and $\varphi_{\beta}\left(a^{*}\right)=\varphi_{\beta}\left(b^{*}\right)$.

Now consider $\varphi_{\beta}\left(x^{*}\right)$ and $\varphi_{\beta}\left(k_{m}^{*}\right)$. Clearly the net $\left\{\varphi_{\beta}\left(k_{m}^{*}\right)\right\}$ converges to $\varphi_{\beta}\left(z^{*}\right)$ and hence to $\varphi_{\beta}\left(y^{*}\right) ;\left(\varphi_{\beta}\left(x^{*}\right), \varphi_{\beta}\left(k_{m}^{*}\right)\right) \in R\left(\varphi_{\beta-1}^{\beta}\right) ;$ and $\lim \left(\varphi_{\beta}\left(x^{*}\right), \varphi_{\beta}\left(k_{m}^{*}\right)\right) t_{m}=\left(\varphi_{\beta}\left(a^{*}\right), \varphi_{\beta}\left(a^{*}\right)\right)$. Thus, we must conclude that $\left(\varphi_{\beta}\left(x^{*}\right), \varphi_{\beta}\left(y^{*}\right)\right)$ is in $Q\left(\varphi_{\beta-1}^{\beta}\right)$. Since $\varphi_{\beta}\left(x^{*}\right) \neq \varphi_{\beta}\left(y^{*}\right)$ by construction and $\varphi_{\beta-1}^{\beta}$ is almost periodic, this is a contradiction. We therefore must conclude that $x \notin S R P(K, y)$ completing the proof of the theorem.

The following lemmas contain useful characterizations of the conditions of Theorem 1.1.

Lemma 1.1. Suppose $(X, T)$ is minimal and $K \cong X$ is closed and nontrivial, then the following are equivalent.

(1) There is a $w \in J(T)$ such that $K w \cap K$ is dense in $K$.

(2) There is a cartesian product $X_{\alpha}\left(X_{\alpha}, T\right)$ where $\left(X_{\alpha}, T\right)=(X$, T) for each $\alpha$ and a point $\left\langle x_{\alpha}\right\rangle$ in $\mathrm{X}_{\alpha} X_{\alpha}$ such that

(a) the range of $\left\langle x_{\alpha}\right\rangle$, that $i s,\left\{x_{\alpha}\right\}$, is a dense subset of $K$, and

(b) $\left\langle x_{\alpha}\right\rangle$ is an almost periodic point of $\mathrm{X}_{\alpha}\left(X_{\alpha}, T\right)$. 
The proof is straightforward and is omitted.

Lemma 1.2. Suppose $(X, T)$ is minimal. Suppose $K \cong X$ and $x \in K$, then the following are equivalent.

(1) $x \in \operatorname{SRP}(K, y)$ for all $y \in K$.

(2) For every index $\alpha$ for $X, \alpha T(x) \cap K$ is dense in $K$.

The proof involves the iterated limit theorem for nets and is also omitted.

As an application, combining Theorem 1.1, Lemma 1.2, and the general Furstenberg structure theorem for distal flows [3], we have the following characterization for distal flows.

THEOREM 1.2. A minimal flow $(X, T)$ is distal iff for every nontrivial closed $K$ and for every $x \in K$, there is an index $\alpha$ such that $\alpha T(x) \cap K$ is not dense in $K$.

Proof. Suppose $(X, T)$ is distal. By the general Furstenberg structure theorem, $(X, T)$ is PI. Then, by Theorem 1.1 and Lemma 1.2 , for every $w \in J$ and every nontrivial $K$ such that $K=c(K w)$, and for every $x \in K$, there is an index $\alpha$ such that $\alpha T(x) \cap K$ is not dense in $K$. Since $(X, T)$ is distal, $K=K w$ for all $w \in J$, completing the first half of the proof.

Suppose for every nontrivial closed $K$ and for every $x \in K$ there is an index $\alpha$ such that $\alpha T(x) \cap K$ is not dense in $K$. We show that every pair of distinct points are distal. Let $x, y \in X$ and let $K=\{x, y\}$. Then either $x=y$ or there is an index $\alpha$ such that $\alpha T(x) \cap K=\{x\}$. Thus, either $x=y$ or $x$ and $y$ are not proximal; $(X, T)$ is distal.

2. The Veech structure theorem. In a recent paper [3] Ellis proves that all distal minimal sets satisfy the Furstenberg structure theorem, that is, all distal minimal sets are PI. Using a modification of Ellis's technique and Theorem 1.1, we show that, for a large class of properties, all minimal flows with a given one of these properties are PI if and only if all metric minimal flows with the given property are PI. One of these properties is "there is a point with countable proximal cell". It then follows from known results that any flow with a point with countable proximal cell is PI. Since all point-distal flows satisfy this property, it follows that all pointdistal flows are PI.

Suppose $P$ is a property of transformation groups. We will call $P$ a transferable property if

(1) $P$ is preserved by transformation group homomorphisms onto minimal sets, and 
(2) if $(X, T)$ has property $P$ and $S$ is a subgroup of $T$ then there is a point $x^{*} \in X$ such that $\left(c\left(x^{*} S\right), S\right)$ has property $P$.

As will be seen in the proof of the main theorm of this section (Theorem 2.1), conditions (1) and (2) are what is needed to transfer property $P$ from a minimal $(X, T)$ to a constructed minimal metric $(Y, H)$.

For the purposes of the following discussion we assume $(X, T)$ is minimal with a transferable property $P$ and that $(X, T)$ is not a PI flow. Then by Theorem 1.1 and Lemma 1.2 there is a nontrivial closed subset $K \cong X$, an idempotent $u \in J(T)$, and an $x_{0} \in K$ such that $c(K u)=K$ and $\alpha T\left(x_{0}\right) \cap K$ is dense in $K$ for every index $\alpha$ on $X$. We assume that $d$ is a continuous pseudo-metric for $X$ for which $\sup \left\{d\left(x_{0}, x\right) \mid x \in K\right\} \neq 0$ and for which $d(x, y) \leqq 1$ for all $x, y \in$ $X$. Proofs of some of the following can be found in [3].

Suppose $H$ is a countable subgroup of $T$. Define $R(H)$ to be $\left\{\left(x_{1}, x_{2}\right) \in X \times X \mid d\left(x_{1} h, x_{2} h\right)=0\right.$ for all $\left.h \in H\right\}$. Then $R(H)$ is a closed, $H$ invariant, equivalence relation. We denote the quotient map from $X$ to $X / R(H)$ by $\varphi$. Suppose $H$ is indexed by $H=\left\{h_{i}\right\}_{i=1}^{\infty}$. For every $a, b \in X / R(H)$ let $\sigma(a, b)=\sum_{i=1}^{\infty} 2^{-i} d\left(x_{1} h_{i}, x_{2} h_{i}\right)$ where $\varphi\left(x_{1}\right)=$ $a$ and $\varphi\left(x_{2}\right)=b$. Then $\sigma$ is a metric whose topology is the quotient topology on $X / R(H)$. Thus $X / R(H)$ is a compact metric space and is therefore 2 nd countable. We let $\mathscr{B}=\mathscr{B}(H)$ be a countable basis for the topology of $X / R(H)$. If $\left\{H_{\imath}\right\}_{i=1}^{\infty}$ is a sequence of countable subgroups of $T$ such that $H_{\imath} \subseteq H_{i+1}$ and $H=\bigcup_{i=1}^{\infty} H_{i}$ and if for $i \leqq$ $j, \psi_{i}^{j}: X / R\left(H_{j}\right) \rightarrow X / R\left(H_{\imath}\right)$ is the cannonical map, then $X / R(H)=\operatorname{inv}$ $\lim \left\{X / R\left(H_{i}\right), \psi_{i}^{j}\right\}$.

The basic idea is that used by Ellis in Proposition 1.6 of [3]. We would like to find a subgroup $H$ of $T$ so that (a) $(X / R(H), H)$ is metric; (b) $\varphi\left(x_{0}\right)$ is an almost periodic point with dense orbit; (c) $\varphi(K)=K^{*}$ has the properties which insure that $(X / R(H), H)$ is not a PI flow; and (d) $(X / R(H), H)$ is a $P$-flow. In general we can insure (a) by choosing $H$ countable and (d) comes for free if we have (b). If $H$ is any countable subgroup of $T$ we can find another countable subgroup $H^{\prime}, H \subseteq H^{\prime}$, so that, relative to the new group $H^{\prime}$, (b) and (c) are true in $X / R(H)$. Since, in general, $\left(X / R(H), H^{\prime}\right)$ is not a transformation group, it is necessary to use an induction and pass to a limit. Before stating the induction lemma we need to introduce some notation.

To show that $\varphi\left(x_{0}\right) \in \varphi(X)=Y$ is an almost periodic point, for any subgroup $H$ of $T$, and any $V \in \mathscr{B}(H)$, we will denote by $F_{V}$ a finite subset of $T$ with the property that $x_{0} T \subseteq \varphi^{-1}[V] F_{V}$. The existence of /these finite sets is guarenteed by the fact that $x_{0}$ is almost periodic in $X$.

To show (c), that $(X / R(H), H)$ is not a PI flow, we use Lemma 
1.1 and Theorem 1.1. We would like to show the existence of a $\left\langle y_{n}\right\rangle \in \mathbf{X}_{i=1}^{\infty} Y$ that is almost periodic and has dense range $\left\{y_{n}\right\}$ in $K^{*}$. In actuality we are forced into $X_{i=1}^{\infty}\left(X_{j=1}^{\infty} Y\right)$ in order to index things properly. We do this by inductively constructing $\left\langle\left\langle y_{n}\right\rangle_{m}\right\rangle_{\text {. }}$ Suppose $H$ is a subgroup of $T$ and suppose we have $K_{n}=\left\{k_{i, j} \mid 1 \leqq\right.$ $j \leqq n, 1 \leqq i<\infty\}$ a subset of $K u$. Let $\mathscr{B}_{n}$ be the basis for $X_{j=1}^{n}\left(\mathbf{X}_{i=1}^{\infty} X\right)$ $R(H)$ ) formed from $\mathscr{B}(H)$. Let $\left\langle\left\langle k_{i}\right\rangle_{j}\right\rangle$ be the element of $\mathbf{X}_{j=1}^{n}\left(\mathbf{X}_{i=1}^{\infty} X\right)$ whose $j$ th coordinate has $k_{i, j}$ as its $i$ th coordinate. Then $\left\langle\left\langle k_{i}\right\rangle_{j}\right\rangle$ is an almost periodic point of $X_{j=1}^{n}\left(X_{i=1}^{\infty} X\right)$ since $\left\langle\left\langle k_{i}\right\rangle_{j}\right\rangle u=\left\langle\left\langle k_{i}\right\rangle_{j}\right\rangle$. Then, for each $U \in \mathscr{B}_{n}$, we will denote by $E_{U}$ a finite subset of $T$ such that $\left\langle\left\langle k_{i}\right\rangle_{j}\right\rangle T \subseteq \hat{\varphi}^{-1}[U] E_{U}$ where $\hat{\varphi}$ is the map from $X_{j=1}^{n}\left(X_{i=1}^{\infty} X\right)$ into $\mathbf{X}_{j=1}^{n}\left(\mathbf{X}_{i=1}^{\infty} X / R(H)\right)$ induced by $\varphi$.

LEMmA 2.1. Suppose $H$ is a countable subgroup of $T$ and $K(n)=\left\{k_{i, j} \mid 1 \leqq j \leqq n, 1 \leqq i<\infty\right\} \subseteq K u$. Then there is a countable subgroup $H^{*}$ of $T$ and $a$ set $K(n+1)=\left\{k_{i, j}^{*} \mid 1 \leqq j \leqq n+1,1 \leqq\right.$ $i<\infty\} \subseteq K u$ such that

(1) $H \subseteq H^{*}$.

(2) $k_{i, j}^{*}=k_{i, j}$ for $1 \leqq j \leqq n, 1 \leqq i<\infty$.

(3) $\varphi\left(x_{0} H^{*}\right)$ is dense in $X / R(H)$.

(4) $\varphi(K(n+1))$ is dense in $\varphi(K)$.

(5) For any $V \in \mathscr{B}(H), \varphi\left[\left(\varphi^{-1}[V] \times \varphi^{-1}[V]\right) H^{*}\left(x_{0}\right) \cap K\right]$ is dense in $\varphi(K)$.

(6) $\cup\left\{F_{V} \mid V \in \mathscr{B}(H)\right\} \subseteq H^{*}$.

(7) $\cup\left\{E_{U} \mid U \in \mathscr{B}_{n+1}(H)\right\} \subseteq H^{*}$.

Proof. For each $V \in \mathscr{B}(H)$ let $t_{V} \in T$ such that $x_{0} t_{V} \in \varphi^{-1}[V]$ and let $L_{1}=\left\{t_{V} \mid V \in \mathscr{B}(H)\right\}$. Then $\varphi\left(x_{0} L_{1}\right)$ is dense in $\varphi(X)$ and $L_{1}$ is countable.

For each $W \in \mathscr{B}(H)$ such that $K \cap \varphi^{-1}[W] \neq \phi$ and for each $V \in \mathscr{B}(H)$ let $t=t(V, W)$ be such that $\left[\left(\varphi^{-1}[V] \times \varphi^{-1}[V]\right) t\right]\left(x_{0}\right) \cap$ $K \cap \mathscr{\varphi}^{-1}[W] \neq \phi$. Let $L_{2}=\left\{t(V, W) \mid K \cap \varphi^{-1}[W] \neq \phi, V, W \in \mathscr{B}(H)\right\}$. Then $\left.\varphi\left[\left(\varphi^{-1}[V] \times \varphi^{-1}[V]\right) L_{2}\right)\left(x_{0}\right) \cap K\right]$ is dense in $\varphi(K)$ and $L_{2}$ is countable.

Let $L_{3}=U\left\{F_{V} \mid V \in \mathscr{B}(H)\right\}$. Then $L_{3}$ is countable.

Since $\varphi(K u)$ is dense in $\varphi(K)$ we can choose a countable subset, $K^{\prime}$, of $K u$ such that $\varphi\left(K^{\prime}\right)$ is dense in $\varphi(K)$. Let $K^{\prime}$ be indexed by $K^{\prime}=\left\{k_{i}^{\prime}\right\}_{i=1}^{\infty}$. Let $\left.\left\langle k_{i}^{*}\right\rangle_{j}\right\rangle$ be the element of $X_{j=1}^{n+1}\left(X_{i=1}^{\infty} X\right)$ whose $j$ th coordinate, $1 \leqq j \leqq n$, has $k_{i, j}$ as its $i$ th coordinate and whose $(n+1)$ th coordinate has $k_{i}^{\prime}$ as its $i$ th coordinate. Then $\left.\left\langle k_{i}^{*}\right\rangle_{j}\right\rangle$ is an almost periodic point of $X_{j=1}^{n+1}\left(X_{i=1}^{\infty} X\right)$. For each $U \in \mathscr{B}_{n+1}(H)$ choose $E_{U}$ as described above. Let $K(n+1)=K(n) \cup K^{\prime}$ with the obvious indexing. Let $L_{4}=\cup\left\{E_{U} \mid U \in \mathscr{B}_{n+1}(H)\right\}$. Then $L_{4}$ is countable.

Let $H^{*}$ be the subgroup of $T$ generated by $H \cup L_{1} \cup L_{2} \cup L_{3} \cup$ $L_{4}$. Then, since each of these sets is countable, $H^{*}$ is countable. 
It is now easily checked that $K(n+1)$ and $H^{*}$ satisfy the requirements of the lemma.

THEOREM 2.1. Suppose $P$ is a transferable property of flows. Then every minimal P-flow is a PI flow iff every metric minimal $P$-flow is a PI flow.

Proof. Clearly if every minimal $P$-flow is a PI flow then every metric minimal $P$-flow is a PI flow.

Suppose $(X, T)$ is a minimal $P$-flow and $(X, T)$ is not PI. Then, from Theorem 1.1 and Lemma 1.2, there is a nontrivial closed set $K \subseteq X$, a point $x_{0} \in X$, and an idempotent $u \in J(T)$, such that $c(K u)=$ $K$ and $\alpha T\left(x_{0}\right) \cap K$ is dense in $K$ for all indices $\alpha$ on $X$. Choose a pseudo-metric $d$ as in the above discussion.

Let $k_{1} \in K u, K_{1}=\left\{k_{1}\right\}$ and $\left\langle k_{i, 1}\right\rangle \in X_{i=1}^{\infty} X$ so that $k_{i, 1}=k_{1}$. Let $H_{1}=\{e\}$ where $e$ is the group identity. Using Lemma 2.1 construct a countable subgroup $H_{2} \subseteq T$ and a countable set $K_{2}=\left\{k_{i, j} \mid j=1,2\right.$; $1 \leqq i<\infty\} \leqq K u$ satisfying the lemma. Proceed by induction to construct sequences $\left\{H_{n}\right\}_{n=1}^{\infty}$ and $\left\{K_{n}=\left\{k_{i, j} \mid 1 \leqq j \leqq n, 1 \leqq i<\infty\right\}\right\}$ such that $H_{n} \subseteq H_{n+1}$ for $n \geqq 1, K_{n} \subseteq K_{n+1}$ for $n \geqq 1$, and for each $n$, letting $H=H_{n}, K(n)=K_{n}, H^{*}=H_{n+1}$ and $K(n+1)=K_{n+1}$, the conditions of Lemma 2.1 are satisfied. For each $n$, let $X / R\left(H_{n}\right)=$ $X_{n}, \varphi_{n}: X \rightarrow X_{n}$. For $i \leqq j$ let $\psi_{i}^{j}: X_{j} \rightarrow X_{i}$ be the map induced by $H_{i} \subseteq H_{j}$. Let $Y=\operatorname{inv} \lim \left\{X_{n}, \psi_{i}^{j}\right\}$ and $H=\bigcup_{n=1}^{\infty} H_{n}$. Then $(Y, H)$ is a transformation group, $Y$ is a compact metric space, and $(Y, H)$ is $(X / R(H), H)$. Let $\varphi: X \rightarrow Y$ be the quotient map and $\pi_{n}: Y \rightarrow X_{n}$ the projection map, $n \geqq 1$.

\section{REMARK 1. $(Y, H)$ is minimal.}

We prove this remark by showing that $y_{0}=\varphi\left(x_{0}\right)$ is a transitive point which is almost periodic. The collection of sets of the form $\pi_{n}^{-1}\left[V_{n}\right]$ where $n$ ranges over the positive integers and $V_{n}$ ranges over $\mathscr{B}_{n}$ is a basis for $Y$ (see [2]). Let $\pi_{n}^{-1}\left[V_{n}\right]$ be a basic open set. Since $\varphi_{n}\left(x_{0} H_{n+1}\right)$ is dense in $X_{n}$, there is a $t \in H_{n+1}$ such that $\varphi_{n}\left(x_{0} t\right) \in V_{n}$. But, since $H_{n+1} \subseteq H, \varphi\left(x_{0} t\right) \in \varphi\left(x_{0}\right) H \cap \pi_{n}^{-1}\left[V_{n}\right]$ and $y_{0}$ is transitive.

To see that $y_{0}$ is almost periodic, let $V_{n} \in \mathscr{B}\left(H_{n}\right)$. We claim $y_{0} H \leqq \pi_{n}^{-1}\left[V_{n}\right] F_{V_{n}}$. Indeed, by construction $x_{0} T \leqq \varphi_{n}^{-1}\left[V_{n}\right] F_{V_{n}}$; so for $h \in H, x_{0} h \in \varphi_{n}^{-1}\left[V_{n}\right] F_{V_{n}}$ and thus $\varphi\left(x_{0} h\right) \in \varphi\left[\varphi_{n}^{-1}\left[V_{n}\right] F_{V_{n}}\right]=\varphi\left[\varphi_{n}^{-1}\left[V_{n}\right]\right] F_{V_{n}}=$ $\pi_{n}^{-1}\left[V_{n}\right] F_{V_{n}}$. Now since $F_{V_{n}} \subseteq H_{n+1} \subseteq H$ and $F_{V_{n}}$ is finite, $y_{0}$ is almost periodic. 
Since $P$ is a transferable property there is an $x^{*} \in X$ such that $\left(c\left(x^{*} H\right), H\right)$ is a $P$-flow, and since $(Y, H)$ is minimal, $\varphi:\left(c\left(x^{*} H\right), H\right) \rightarrow$ $(Y, H)$ is surjective. Since $\left(c\left(x^{*} H\right), H\right)$ is a $P$-flow and $P$ is transferable, $(Y, H)$ is a $P$-flow.

REMARK 3. Let $K^{\prime}=\bigcup_{n=1}^{\infty} K_{n}$. Then $\varphi\left(K^{\prime}\right)$ is dense in $\varphi(K)$. We leave the proof of this remark to the reader.

REMARK 4. There is an idempotent $u^{*} \in J(H)$ such that $\varphi\left(K^{\prime}\right) u^{*}=$ $\varphi\left(K^{\prime}\right)$.

Define $\left\langle\left\langle a_{i}\right\rangle_{j}\right\rangle \in \mathbf{X}_{j=1}^{\infty}\left(\mathbf{X}_{i=1}^{\infty} Y\right)$ by $a_{i, j}=\varphi\left(k_{i, j}\right) \in \varphi\left(K^{\prime}\right)$. By Lemma 1.1, we can complete the proof of this remark by demonstrating that $\left.\left\langle a_{\imath}\right\rangle_{j}\right\rangle$ is almost periodic in $\mathbf{X}_{j=1}^{\infty}\left(\mathbf{X}_{i=1}^{\infty} Y\right)$.

A basic open set in $\mathbf{X}_{j=1}^{\infty}\left(\mathbf{X}_{i=1}^{\infty} Y\right)$ is of the form $f_{n}^{-1}\left[V_{n}\right]$ where $f_{n}: \mathbf{X}_{j=1}^{\infty}\left(\mathbf{X}_{i=1}^{\infty} Y\right) \rightarrow \mathbf{X}_{j=1}^{n}\left(\mathbf{X}_{i=1}^{\infty} X_{n}\right)$ is the obvious projection map and $V_{n}$ is a basic open set in $\mathbf{X}_{j=1}^{n}\left(\mathbf{X}_{i=1}^{\infty} X_{n}\right)$. By construction, for every such $V_{n},\left\langle\left\langle k_{i}\right\rangle_{j}\right\rangle T \leqq \hat{\varphi}_{n}^{-1}\left[V_{n}\right] E_{V_{n}}$ where $E_{V_{n}} \leqq H, 1 \leqq i<\infty, 1 \leqq j \leqq n$, and $\hat{\varphi}_{n}: \mathbf{X}_{j=1}^{n}\left(\mathbf{X}_{i=1}^{\infty} X\right) \rightarrow \mathbf{X}_{j=1}^{n}\left(\mathbf{X}_{i=1}^{\infty} X_{n}\right)$ is the homomorphism induced by $\varphi_{n}$. Let $g_{n}$ be the projection from $\mathbf{X}_{j=1}^{\infty}\left(\mathbf{X}_{i=1}^{\infty} X\right)$ onto $\mathbf{X}_{j=1}^{n}\left(\mathbf{X}_{i=1}^{\infty} X\right)$. Then, $\left.\left\langle\left\langle k_{i}\right\rangle_{j}\right\rangle H \leqq\left\langle k_{i}\right\rangle_{j}\right\rangle T \subseteq g_{n}^{-1} \hat{\varphi}_{n}^{-1}\left[V_{n}\right] E_{V_{n}}, 1 \leqq i<\infty, 1 \leqq j<\infty$. Therefore, $\left\langle\left\langle a_{i}\right\rangle_{j}\right\rangle H \subseteq f_{n}^{-1}\left[V_{n}\right] E_{V_{n}}$ and since $E_{V_{n}}$ is finite and a subset of $H,\left\langle\left\langle a_{2}\right\rangle_{j}\right\rangle$ is almost periodic, completing the proof of Remark 4.

REMark 5. For every index $\beta$ on $Y, \beta H\left(y_{0}\right) \cap \varphi(K)$ is dense in $\varphi(K)$.

Let $\beta$ be an index on $Y$. Then there is a basic open set $\pi_{n}^{-1}\left[V_{n}\right]$ such that $\left(\pi_{n}^{-1}\left[V_{n}\right] \times \pi_{n}^{-1}\left[V_{n}\right]\right) H \subseteq \beta H$. Let $\pi_{m}^{-1}\left[W_{m}\right]$ be any basic open set for which $\pi_{m}^{-1}\left[W_{m}\right] \cap \varphi(K) \neq \phi$. Let $n^{*}=\max (m, n)$ and let $V_{n^{*}}=$ $\left(\psi_{n}^{n^{*}}\right)^{-1}\left[V_{n}\right]$ and $W_{n^{*}}=\left(\gamma_{m}^{n^{*}}\right)^{-1}\left[W_{m}\right]$. Then $\pi_{m}^{-1}\left[W_{m}\right]=\pi_{n^{*}}^{-1}\left[W_{n^{*}}\right]$ and $\pi_{n}^{-1}\left[V_{n}\right]=\pi_{n^{*}}^{-1}\left[V_{n^{*}}\right]$. By construction, $\varphi_{n^{*}}\left[\left(\varphi_{n^{*}}^{-1}\left[V_{n^{*}}\right] \times \varphi_{n^{*}}^{-1}\left[V_{n^{*}}\right]\right) H_{n^{*}}\left(x_{0}\right) \cap\right.$ $K] \cap W_{n^{*}}$ is not empty, say it contains $\varphi\left(x^{*}\right)$ where $x^{*} \in\left(\varphi_{n^{*}}^{-1}\left[V_{n^{*}}\right] \times\right.$ $\left.\varphi_{n^{*}}^{-1}\left[V_{n^{*}}\right]\right) H_{n^{*}}\left(x_{0}\right) \cap K$. Then, $\varphi\left(x^{*}\right)$ is in $\pi_{n^{*}}^{-1}\left[W_{n^{*}}\right]$ and in $\varphi(K)$. There is an $h \in H_{n^{*}}$ such that $\left(x^{*}, x_{0}\right) h^{-1} \in \varphi_{n^{*}}^{-1}\left[V_{n^{*}}\right] \times \varphi_{n^{*}}^{-1} /\left[V_{n^{*}}\right]$ and hence $\left(\varphi\left(x^{*}\right)\right.$, $\left.y_{0}\right) h^{-1} \in \pi_{n^{*}}^{-1}\left[V_{n}\right] \times \pi_{n^{*}}^{-1}\left[V_{n}\right]$. Since $h \in H_{n^{*}} \subseteq H, \beta H\left(y_{0}\right) \cap \varphi(K) \cap \pi_{m}^{-1}\left[W_{m}\right]$ contains $\varphi\left(x^{*}\right)$. Thus, $\beta H\left(y_{0}\right) \cap \varphi(K)$ is dense in $\varphi(K)$.

Remark 6. Combining Remarks $1,3,4$, and 5 we conclude that, since $K^{*}$ is nontrivial by the original choice of $d,(Y, H)$ is not PI. By Remark 2, $(Y, H)$ is a $P$-flow. Thus, if there is a non-PI minimal $P$-flow there is a non-PI metric minimal $P$-flow, completing the proof of the theorem.

It is well known that any metric minimal flow that contains a point $x_{0}$ for which $P\left[x_{0}\right]=\left\{x \in X \mid x\right.$ is proximal to $\left.x_{0}\right\}$ is countable is a PI flow $[5,6]$. In particular, if $(X, T)$ has a distal point this 
is the PI version of the Veech structure theorem. (The HPI version states that point-distal flows which are quasi-separable are HPI, the proximal maps in the PI tower are highly-proximal [1].) The property "has a point whose proximal cell is countable" is transferable and thus, by the above theorem and known results, any minimal flow with this property is PI. Thus, the PI version of the Veech structure theorem holds in general. We will also show that the HPI version holds in general.

Lemma 2.2. The property: $(X, T)$ contains a point $x_{0}$ such that $P\left[x_{0}\right]=\left\{x \in X \mid x\right.$ is proximal to $\left.x_{0}\right\}$ is countable; is transferable.

Proof. Suppose $(X, T)$ has this property. Let $\varphi:(X, T) \rightarrow(Y$, $T$ ) be a transformation group homomorphism and $(Y, T)$ be minimal. Then $\varphi\left(P\left[x_{0}\right]\right)=P\left[\varphi\left(x_{0}\right)\right]$ and hence $(Y, T)$ has the property.

Suppose $S$ is a subgroup of $T$. Consider $\left(c\left(x_{0} S\right), S\right)$. Clearly if $y$ is $S$-proximal to $x_{0}$ then $y$ is $T$-proximal to $x_{0}$ and hence $\left(c\left(x_{0} S\right), S\right)$ has the property. Thus it is transferable.

Corollary 2.1. Any minimal set that has a point with countable proximal cell is PI.

Proof. Use X.7.2 of [6], Theorem 2.1, and Lemma 2.2.

COROLLARY 2.2. (General Veech structure theorem-PI Version) A point-distal flow is PI.

Note that Corollary 2.1 also implies the general Furstenberg structure theorem. We conclude this paper by proving the HPI version of the general Veech structure theorem. The following lemma is needed.

Lemma 2.3. Suppose $(X, T)$ and $(Y, T)$ are minimal and $\varphi$ : $(X, T) \rightarrow(Y, T)$ is a homomorphism. Suppose $(X, T)$ is PI and $D(\varphi)$ is dense in $R(\varphi)$. Then either $\varphi$ is an isomorphism or $S(\varphi) \neq R(\varphi)$.

Proof. Fix $u \in J$, and let $x_{0}, y_{0}$ be points in $X$ and $Y$ respectively such that $x_{0} u=x_{0}, y_{0} u=y_{0}$, and $\varphi\left(x_{0}\right)=y_{0}$. Since $D(\varphi)$ is dense in $R(\varphi), \Phi$ is full by 4.3 of [5]. Thus there exists a flow $Z$ such that the diagram

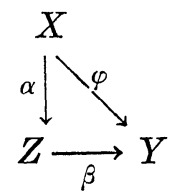


commutes, $\beta$ is almost periodic, and $G(Z)=G(X) H(G(Y)$ ) where $G(X)=G\left(X, x_{0}\right), G(Y)=G\left(Y, y_{0}\right), G(Z)=G\left(Z, \alpha\left(x_{0}\right)\right)$ and $H$ is as defined in 1.9 of [6], page 117.

Now suppose $S(\varphi)=R(\varphi)$. Then $\beta$ is an isomorphism and thus $G(Y)=G(X) H(G(Y))$. For each ordinal $\lambda$ let $H_{\lambda+1}(G(Y))=H\left(H_{\lambda}(G(Y))\right)$ and if $\lambda$ is a limit ordinal then $H_{\lambda}(G(Y))=\cap\left\{H_{\gamma}(G(Y)): \gamma<\lambda\right\}$. It then follows by induction and X.4.1 of [6] that $G(Y)=G(X) H_{\lambda}(G(Y)$ ) for all ordinals $\lambda$. Let $\eta$ be the least ordinal for which $G_{\eta}=G_{\infty}$. Then $H_{\eta}(G(Y)) \subseteq G_{\infty}$. We then have

$$
G(Y)=G(X) H_{\eta}(G(Y)) \cong G(X) G_{\infty}=G(X)
$$

since $(X, T)$ is $\mathrm{PI}$.

Therefore $G(Y)=G(X)$ and $\varphi$ is proximal. Thus $D(\varphi) \cong P(\varphi)$ and hence, since $D(\varphi)$ is dense in $R(\varphi), R(\varphi)=\Delta$ and $\varphi$ is an isomorphism.

Suppose $(X, T)$ is a PI flow. To construct the cannonical PI tower for $(X, T)$ we begin with the map $X \rightarrow\{x\}$ from $X$ to the one point flow, and use the prime construction $[5,6]$ to form the diagram

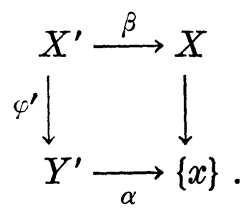

Since $(X, T)$ is $\mathrm{PI}$, either $\varphi^{\prime}$ is an isomorphism or $S\left(\varphi^{\prime}\right) \neq R\left(\varphi^{\prime}\right)$ and a nontrivial $Z^{\prime}$ can be inserted so that the following diagram commutes.

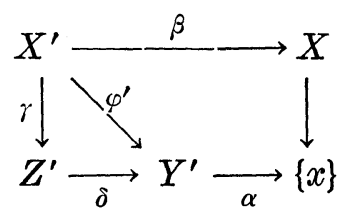

The prime construction is then applied to $\gamma$. The process, inductively, yeilds a PI tower for $X$.

An alternative to the prime construction of Ellis, Glasner, and Shapiro is the star construction originally due to Veech [8] (see also [9]). In general, the star construction yields a diagram

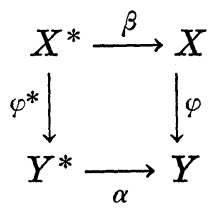


where $\alpha$ and $\beta$ are highly proximal and $\varphi^{*}$ is open, but in general $S\left(\varphi^{*}\right)$ may be equal to $R\left(\varphi^{*}\right)$ without $\phi^{*}$ being an isomorphism. When the star construction can be used, the constructed tower is an HPI tower [1]. We show that for point-distal flows the star construction can be used to construct an HPI tower and hence point-distal flows are HPI.

THEOREM 2.2. (General Veech structure theorem-HPI version) Every point-distal flow is HPI.

Proof. Recall that if $\varphi: X \rightarrow Y$ then $x_{0} \in X$ is a $\varphi$-distal point iff $\varphi(x)=\varphi\left(x_{0}\right)$ implies $x$ and $x_{0}$ are distal. We first note that if $\varphi: X \rightarrow Y$ is open and $x_{0}$ is a $\varphi$-distal point, then $D(\varphi)$ is dense in $R(\varphi)$, and that if $\varphi: X \rightarrow Y$ has $x_{0}$ as a $\varphi$-distal point then $\varphi^{*}: X^{*} \rightarrow$ $Y^{*}$ has a $\varphi^{*}$ distal point (see, for example, [4] for proofs).

We then proceed by transfinite induction, using pointed flows, to construct a diagram

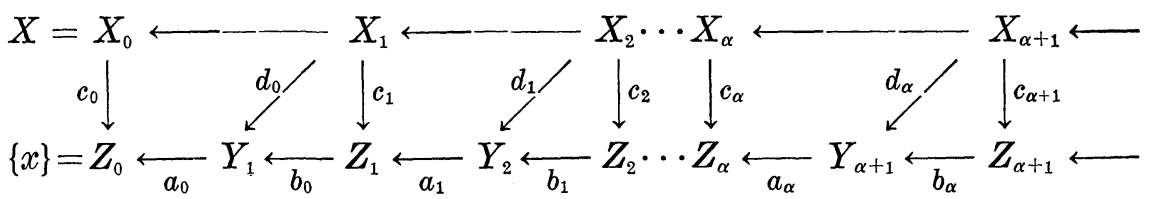

where for any nonlimit ordinal $i, X_{i}=X_{i-1}^{*}, Y_{i}=Z_{i-1}^{*}, d_{i}=c_{i-1}^{*}$. The existence of $Z_{i}, b_{i-1}$, and $c_{i}$ are guarenteed by the remarks above, the fact that $X$ is point-distal and hence PI by Corollary 2.2, and by Lemma 2.3. If $i$ is a limit ordinal $X_{i} \stackrel{c_{i}}{\rightarrow} Z_{i}$ is the inverse limit of the diagram for all $\lambda<i$ and hence $X_{i} \stackrel{c_{i}}{\rightarrow} Z_{i}$ will have a $c_{\imath}$ distal point. If $\eta$ is the least ordinal for which $R\left(d_{\eta}\right)=S\left(d_{\eta}\right)$, then $d_{\eta}$ is an isomorphism and the diagram yields an HPI tower for $X$, completing the proof.

\section{REFERENCES}

1. J. Auslander and S. Glasner, Distal and highly proximal extensions of minimal flows, Univ. of Maryland, (preprint).

2. J. Dugundji, Topology, Allyn and Bacon, Boston, 1968.

3. R. Ellis, The Fustenberg structure theorem, Pacific J. Math., 76 (1978), 345-349.

4. - The Veech structure theorem, TAMS, 186 (1973), 203-218.

5. R. Ellis, S. Glasner and L. Shapiro, PI Flows, Advances in Math.,

6. S. Glasner, Proximal Flows, Lecture Notes in Math., vol. 517, Springer-Verlag, Berlin and New York, 1976.

7. D. McMahon, Relativized weak disjointness and relatively invariant measures. TAMS, 236 (1978), 225-237.

8. W. A. Veech, Point-distal flows, Amer. J. Math., 92 (1970), 205-242.

9. — Topological Dynamics, BAMS, 83 (1977), 775-830. 
10. I. U. Bronstein, A characteristic property of PD-extensions (Russian), Bul. Akad. Stiince RSS Moldoven, 93 (1977), 11-15.

11. D. McMahon, T. S. Wu, On proximal and distal extensions of minimal sets, Bull. Inst. Math. Sinica, 21 (1974), 93-107.

12. P. Shoenfeld, Highly proximal and generalized almost finite extensions of minimal sets, Pacific J. Math., 1 (1976), 263-288.

Received August 9, 1978 and in revised form June 14, 1979.

ARIzona State University

TEMPE, AZ 85281

AND

OAKLAND UnIVERSITY

ROCHESTER, MI 48063 



\section{PACIFIC JOURNAL OF MATHEMATICS}

\section{EDITORS}

DONALD BABBITT (Managing Editor)

University of California

Los Angeles, CA 90024

HUGo RossI

University of Utah

Salt Lake City, UT 84112

C. C. MOORE and ANDREW OGG

University of California

Berkeley, CA 94720
J. DugundJI

Department of Mathematics

University of Southern California

Los Angeles, CA 90007

R. FinN and J. Milgram

Stanford University

Stanford, CA 94305

ASSOCIATE EDITORS
E. F. BECKENBACH
B. H. NeUmanN
F. WOLF
K. YoSHIDA

\section{SUPPORTING INSTITUTIONS}

UNIVERSITY OF BRITISH COLUMBIA

CALIFORNIA INSTITUTE OF TECHNOLOGY

UNIVERSITY OF CALIFORNIA

MONTANA STATE UNIVERSITY

UNIVERSITY OF NEVADA, RENO

NEW MEXICO STATE UNIVERSITY

OREGON STATE UNIVERSITY

UNIVERSITY OF OREGON
UNIVERSITY OF SOUTHERN CALIFORNIA

STANFORD UNIVERSITY

UNIVERSITY OF HAWAII

UNIVERSITY OF TOKYO

UNIVERSITY OF UTAH

WASHINGTON STATE UNIVERSITY

UNIVERSITY OF WASHINGTON

The Supporting Institutions listed above contribute to the cost of publication of this Journal, but they are not owners or publishers and have no responsibility for its content or policies.

Mathematical papers intended for publication in the Pacific Journal of Mathematics should be in typed form or offset-reproduced, (not dittoed), double spaced with large margins. Please do not use built up fractions in the text of the manuscript. However, you may use them in the displayed equations. Underline Greek letters in red, German in green, and script in blue. The first paragraph or two must be capable of being used separately as a synopsis of the entire paper. Please propose a heading for the odd numbered pages of less than 35 characters. Manuscripts, in triplicate, may be sent to any one of the editors. Please classify according to the scheme of Math. Reviews, Index to Vol. 39. Supply name and address of author to whom proofs should be sent. All other communications should be addressed to the managing editor, or Elaine Barth, University of California, Los Angeles, California, 90024.

50 reprints to each author are provided free for each article, only if page charges have been substantially paid. Additional copies may be obtained at cost in multiples of 50 .

The Pacific Journal of Mathematics is issued monthly as of January 1966. Regular subscription rate: $\$ 84.00$ a year (6 Vols., 12 issues). Special rato: $\$ 42.00$ a year to individual members of supporting institutions.

Subscriptions, orders for numbers issued in the last three calendar years, and changes of address shoud be sent to Pacific Journal of Mathematics, P.O. Box 969, Carmel Valley, CA 93924, U.S.A Old back numbers obtainable from Kraus Periodicals Co., Route 100, Millwood, NY 10546.

PUBLISHED BY PACIFIC JOURNAL OF MATHEMATICS, A NON-PROFIT CORPORATION

Printed at Kokusai Bunken Insatsusha (International Academic Printing Co., Ltd.). 8-8, 3-chome, Takadanobaba, Shinjuku-ku, Tokyo 160, Japan.

Copyright (C) 1980 by Pacific Jounal of Mathematics Manufactured and first issued in Japan 


\section{Pacific Journal of Mathematics \\ Vol. 89, No. $2 \quad$ June, 1980}

Frank Hayne Beatrous, Jr. and R. Michael Range, On holomorphic

approximation in weakly pseudoconvex domains................. 249

Lawrence Victor Berman, Quadratic forms and power series fields ...... 257

John Bligh Conway and Wacław Szymański, Singly generated antisymmetric operator algebras ....................... 269

Patrick C. Endicott and J. Wolfgang Smith, A homology spectral sequence for submersions . . . .................................

Sushil Jajodia, Homotopy classification of lens spaces for one-relator groups with torsion ................................ 301

Herbert Meyer Kamowitz, Compact endomorphisms of Banach

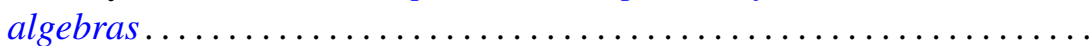

Keith Milo Kendig, Moiré phenomena in algebraic geometry: polynomial

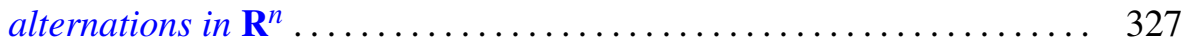

Cecelia Laurie, Invariant subspace lattices and compact operators....... 351

Ronald Leslie Lipsman, Restrictions of principal series to a real form . . . . . 367

Douglas C. McMahon and Louis Jack Nachman, An intrinsic

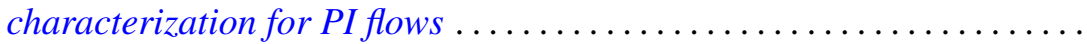

Norman R. Reilly, Modular sublattices of the lattice of varieties of inverse semigroups .................................... 405

Jeffrey Arthur Rosoff, Effective divisor classes and blowings-up of $\mathbf{P}^{2}$ 419

Zalman Rubinstein, Solution of the middle coefficient problem for certain

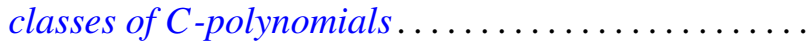

Alladi Sitaram, An analogue of the Wiener-Tauberian theorem for spherical transforms on semisimple Lie groups ................

Hal Leslie Smith, A note on disconjugacy for second order systems ...

J. Wolfgang Smith, Fiber homology and orientability of maps ...

Audrey Anne Terras, Integral formulas and integral tests for series of positive matrices. 